\title{
Criação do Centro Brasileiro de Mindful Eating: resgate da consciência e do amor ao comer*
}

\author{
Ana Paula Leme de Souza ${ }^{1,2}$ \\ (D) https://orcid.org/0000-0003-1257-3394 \\ Fernanda Rodrigues de Oliveira Penaforte ${ }^{3}$ \\ (D) https://orcid.org/0000-0001-8483-1562 \\ Livia Dayane Sousa Azevedo 4 \\ (D) https://orcid.org/0000-0002-0019-9342 \\ Driele Cristina Gomes Quinhoneiro ${ }^{5}$ \\ (D) https://orcid.org/0000-0002-0624-9179 \\ Rosane Pilot Pessa ${ }^{1}$ \\ (D) https://orcid.org/0000-0002-6301-6830
}

\footnotetext{
* Este artigo refere-se à chamada temática "Mindfulness e outras práticas contemplativas".

${ }^{1}$ Universidade de São Paulo, Escola de Enfermagem de Ribeirão Preto, Centro Colaborador da OPAS/OMS para o Desenvolvimento da Pesquisa em Enfermagem, Ribeirão Preto, SP, Brasil.

2 Bolsista da Coordenação de Aperfeiçoamento de Pessoal de Nível Superior (CAPES), Brasil.

${ }_{3}^{3}$ Universidade Federal do Triângulo Mineiro, Departamento de Nutrição, Uberaba, MG, Brasil.

${ }^{4}$ Universidade de São Paulo, Faculdade de Medicina, Ribeirão Preto, SP, Brasil.

${ }^{5}$ Centro Brasileiro de Mindful Eating (CBME), São Paulo, SP, Brasil.
}

Objetivo: descrever a trajetória da criação do Centro Brasileiro de Mindful Eating e buscar compreender as motivações, os objetivos, os resultados já alcançados e as perspectivas e desafios para o futuro dessa organização. Método: estudo descritivo, exploratório com delineamento qualitativo. A coleta de dados foi realizada por meio de consultas aos documentos disponíveis no site do Centro e relatos de profissionais envolvidos em sua idealização e criação. As informações reunidas foram organizadas em categorias temáticas para oferecer descrição consistente do referido Centro enquanto marco referencial para o movimento científico e profissional do comer consciente. Resultados e Discussão: a criação do Centro compreendeu: Organização e estrutura: independência, descentralização e horizontalidade; Objetivos e motivações: amor, cuidado e ética; Desafios e perspectivas futuras: plantar, cuidar e colher. Tais categorias, exploradas em conjunto, revelaram a importância da criação do Centro ao colaborar, entre outras questões, para o estabelecimento do tema no Brasil. Conclusão: o Centro tem colaborado para o desenvolvimento profissional ético dessa área de conhecimento no Brasil, estabelecendo-se no sentido de organizar e oferecer referência para disseminação das referidas práticas. Estudos baseados nessa temática poderão contribuir para que o Mindful Eating se fortaleça enquanto importante recurso para a saúde e qualidade de vida.

Descritores: Atenção Plena; Comportamento Alimentar; Qualidade de Vida; Promoção da Saúde.

\section{Como citar este artigo}

Souza APL, Penaforte FRO, Azevedo LDS, Quinhoneiro DCG, Pessa RP. Creation of the Brazilian Center of Mindful Eating: Recovery of conscience and love when eating. SMAD, Rev Eletrônica Saúde Mental Álcool Drog. 2020;16(3):73-80. doi: https://dx.doi.org/10.11606/issn.1806-6976.smad.2020.167597 


\section{Creation of the Brazilian Center of Mindful Eating: Recovery of conscience and love when eating}

Objective: to describe the trajectory of the creation of the Brazilian Center of Mindful Eating and to attempt to understand the motivations, objectives, the already obtained results, as well as the future perspectives and challenges of this organization. Method: exploratory descriptive study with a qualitative design. Data collection was carried out by consulting the documents available on the Center's website and compiling the accounts given by the professionals involved in conceiving and creating the Center. The obtained information was sorted into categories so as to provide a consistent description of said Center as a milestone for the mindful eating scientific and professional movement. Results and Discussion: creating the Center required: Organization and structure: independence, decentralization and horizontality; Objectives and motivations: love, care and ethics; Challenges and future perspectives: cultivating, taking care of and reaping the benefits of it. Those categories, explored together, have shown how important it was to create the Center to cooperate, among other things, to inaugurate the topic in Brazil. Conclusion: the Center has been contributing to the ethical professional development of this field of knowledge in Brazil and has been establishing itself so as to organize and be the reference to disseminate such practices. Studies based on this subject may contribute to strengthen Mindful Eating as an important resource for health and quality of life.

Descriptors: Mindfulness; Feeding Behavior; Quality of Life; Health Promotion.

\section{Creación del Centro Brasileño de Mindful Eating: el rescate de la conciencia y del amor al comer}

Objetivo: describir la trayectoria de la creación del Centro Brasileño de Mindful Eating y buscar comprender los motivos, los objetivos, los resultados ya alcanzados y las perspectivas y desafíos para el futuro de esta organización. Metodología: estudio descriptivo, exploratorio con enfoque cualitativo. La recolección de datos se realizó a través de consultas a los documentos disponibles en la página web del Centro y relatos de profesionales involucrados en su creación. La información recabada fue organizada en categorías temáticas para ofrecer una descripción consistente de dicho Centro como marco referencial para el movimiento científico y profesional del comer consciente. Resultados y Discusión: la creación del Centro involucró: Organización y estructura: independencia, descentralización y horizontalidad; Objetivos y motivos: amor, cuidado y ética; Desafíos y perspectivas futuras: plantar, cuidar y cosechar. Tales categorías, exploradas en conjunto, han revelado la importancia de la creación del Centro al colaborar, entre otros aspectos, al desarrollo de este tema en Brasil. Conclusión: el Centro ha contribuido al desarrollo profesional y ético de esta área de conocimiento en Brasil, estableciéndose a modo de organizar y servir de referencia para la difusión de tales prácticas. Los estudios con base en este tema podrán ayudar a que el Mindful Eating se fortalezca como un importante recurso para la salud y la calidad de vida.

Descriptores: Atención Plena; Conducta Alimentaria; Calidad de Vida; Promoción de la Salud. 


\section{Introdução}

As práticas meditativas podem atuar como atividades de auto-observação com o intuito de cultivar calma, generosidade, compaixão, concentração, ética e autorregulação da atenção à consciência das experiências imediatas $^{(1)}$. A meditação também pode abranger um grupo de habilidades de complexa regulação da atenção e das emoções podendo ser desenvolvidas em vários contextos, como o cultivo do bem-estar e do equilíbrio emocional(2).

Assim, como uma habilidade da mente, a prática de Mindfulness pode ser desenvolvido por meio da meditação laica(3-4), e pode ser definido como a prática de trazer a atenção para o momento presente de forma intencional, com abertura, curiosidade e aceitação, sem julgamentos da experiência, seja ela agradável ou não(5).

Para os que iniciam as meditações Mindfulness, é comum a orientação da prática da respiração como uma meditação de atenção focada(6). Assim, o exercício frequente pode fazer com que o praticante consiga trazer o foco de sua atenção para o momento presente, e às sensações físicas relacionadas com a respiração. Gradualmente o praticante é estimulado a expandir a atenção para as sensações de todo o corpo, se dando conta das emoções presentes, dos pensamentos e outros processos mentais que podem surgir, além de tratar cada um destes conteúdos com equanimidade, ou seja, sem intenções de análise, desejos ou aversões, ao observá-los em relação ao modo como surgem, se modificam, ou deixam de existir(7-8).

No entanto, também é possível entender e desenvolver Mindfulness enquanto habilidade da mente, como um estado cognitivo fluido que resulta de novas interpretações sobre a situação e o ambiente. Ao estar atento, é possível estar ativamente envolvido no presente e ao contexto da situação, com a possibilidade de elaboração de outras perspectivas. A capacidade de visualizar objetos e situações de várias perspectivas e, dependendo do contexto, observar a possibilidade de mudança de perspectiva, são características do estado cognitivo consciente. Esse estado cognitivo difere das práticas de meditação Mindfulness, embora os estados pós-meditativos possam resultar em atenção no sentido cognitivo(9-10).

Entre as habilidades exercitadas nas práticas de Mindfulness, também é possível identificar o cultivo da metacognição para agir de maneira correta. Nesse sentido, a prática pode trazer atenção ao que se está fazendo a cada momento, e assim ter a possibilidade de enxergar verdadeiramente a origem dos próprios atos.
Ao tomar consciência dos comportamentos, palavras ou pensamentos motivados por sentimentos de ganância, ódio e ilusão, ou bondade e compaixão, o Mindfulness também traz associação com consciência moral e pode se caracterizar como um tipo de intuição ética(11).

No mesmo caminho, o Mindful Eating pode ser entendido como a consciência sem julgamento em relação às sensações físicas e emocionais associadas à alimentação(12), o que inclui estar atento ao sabor do alimento na boca, a consciência dos sinais de fome e saciedade, além das emoções despertadas por meio da alimentação(13). O Mindful Eating oferece a oportunidade de desenvolvimento da autonomia e da permissão para a escolha consciente de qualquer tipo de alimento, sem julgamentos, e valoriza toda experiência envolvida no ato de comer, os efeitos da comida nas sensações físicas e emocionais antes, durante e após a alimentação, ao fazer de cada refeição um momento de autocuidado e bondade consigo próprio(14-15).

Muito embora se trate de uma temática recente, especialmente em âmbito nacional, o interesse em Mindfulness e Mindful Eating tem crescido gradualmente nos últimos anos, com ampliação de pesquisas, do interesse por parte de profissionais de saúde por essa linha de trabalho e também da implementação em programas de saúde(16). Nessa perspectiva, é necessário cautela para que não haja distorções nos conceitos e nos princípios das abordagens centradas em Mindfulness e em Mindful Eating, evitando banalizações ou aplicações equivocadas, com vistas a respeitar a ética e o compromisso com as suas normativas, bem como preservar a diversidade que pode ser abordada por meio da prática laica. Portanto, para que haja desenvolvimento sustentável desse novo campo do saber, é necessário articular as definições, os princípios e as diretrizes do que é e do que não é Mindfulness e Mindful Eating ${ }^{(14-17)}$.

A partir desse cenário, profissionais que atuam na área se uniram em torno de um objetivo comum - o de cuidar do campo do Mindful Eating no Brasil, e fundaram o Centro Brasileiro de Mindful Eating (CBME), com o intuito de facilitar o processo de (re)construção de uma relação harmônica com a comida e com o corpo por aqueles que buscam esse caminho.

\section{Objetivo}

Descrever a trajetória da criação do Centro Brasileiro de Mindful Eating (CBME) e buscar compreender as motivações, os objetivos, os resultados já alcançados e as perspectivas e os desafios para o futuro dessa organização. 


\section{Método}

Trata-se de um estudo descritivo e exploratório com delineamento qualitativo. A pesquisa qualitativa foi escolhida por investigar de forma profunda determinado tema, e se preocupar com o grau de realidade que não pode ser quantificado. Assim, trabalha com o universo de significados, motivos, expectativas, valores e atitudes ao permitir que o pesquisador confira significados a determinado fenômeno de maneira interpretativa e compreensiva(18-19)

Os dados foram coletados por meio de consultas aos documentos disponíveis no site do CBME sobre sua história, seus valores, sua missão e sua organização. Também foram coletados relatos de forma individual por meio de ligação telefônica com os profissionais envolvidos em sua idealização e criação. A entrevista continha questionamentos sobre os objetivos, motivações, dificuldades e perspectivas envolvidas no processo de construção e consolidação do CBME. É válido pontuar que uma das autoras deste artigo é também uma das idealizadoras e atuais diretoras do CBME, com participação ativa nesse processo desde o seu início, o que auxiliou a busca das informações necessárias para responder ao objetivo desse estudo.

Relatos e documentos compuseram o corpus analítico deste estudo. Este corpus foi analisado por meio da análise de conteúdo temática que traz a concepção de tema ligado a uma afirmação à respeito de determinado assunto, e permite inúmeras relações e pode ser graficamente apresentada por meio de uma palavra, de uma frase, de um resumo(20). Esse procedimento dá oportunidade para o entendimento da essência das descrições, cuja frequência ou presença terão um significado para o objeto analítico visado(18). As etapas compreendidas na análise temática são: pré-análise; exploração do material; tratamento dos dados obtidos e interpretação. Na pré-análise, todas as entrevistas foram lidas exaustivamente, o que tem por denominação "leitura flutuante", permitindo que as primeiras impressões emergissem. Após essa etapa, todo o conteúdo de interesse foi organizado dentro dos eixos temáticos, que foram definidos a posteriori. Em seguida, foi realizado o agrupamento das mensagens com características comuns em categorias, a partir deste conteúdo organizado(20).

Para a concretização do trabalho foram contemplados os preceitos emanados da Resolução no 466/12 do Conselho Nacional de Saúde (CNS), na qual se propõe respeitar os princípios da autonomia, beneficência, não-maleficência e justiça/equidade.

\section{Resultados e Discussão}

Os documentos disponibilizados pelas diretoras da organização, as informações do site do CBME e as entrevistas realizadas com três das quatro atuais diretoras do CBME resultaram na identificação de três categorias temáticas que serão descritas e discutidas a seguir: (a) Organização estrutural: independência, descentralização e horizontalidade; (b) Objetivos e motivações: amor, cuidado e ética; (c) Desafios e perspectivas futuras: plantar, cuidar e colher.

\section{Organização estrutural: independência, descentralização e horizontalidade}

O CBME é uma organização independente e sem fins lucrativos, criada em 2016. Além de auxiliar no estabelecimento do Mindful Eating no Brasil, esta organização trouxe consigo o esforço conjunto de cinco profissionais para estruturar um lugar que fosse fonte de apoio tanto para profissionais que desejassem atuar na área, quanto para pessoas que desejassem formas mais sustentáveis e significativas de relacionamento com a comida, com o comer e com o corpo. Ao longo dessa caminhada, o CBME vem se fortalecendo, e atualmente é dirigido por quatro mulheres, uma médica, duas nutricionistas e uma antropóloga.

Semelhante ao observado para criação dos Centros de Atenção Psicossocial, cuja inspiração provém, em grande parte, de experiências realizadas no exterior(21), a criação do CBME também se inspirou na experiência internacional do The Center for Mindful Eating (TCME). Criado em 2006 nos Estados Unidos da América, o TCME também é uma organização sem fins lucrativos que zela para que as informações referentes ao Mindful Eating cheguem de forma correta e acessível para formar profissionais nos princípios e nas práticas da alimentação consciente, por meio da promoção da sabedoria, atenção plena e ação ética na educação, política, pesquisa e saúde relacionadas à alimentação(22).

Inicialmente, o CBME se organizou vinculado a uma instituição pública de ensino superior. Após um breve período, houve mobilização de suas idealizadoras motivadas, entre outras fatores, por suas práticas pessoais de Mindfulness e Mindful Eating, para que este se tornasse uma organização independente, com intuito de construir seu próprio caminho, objetivos, missões e valores. Atualmente, o CBME não possui uma sede física, e se localiza apenas em ambiente virtual.

O CBME também conta com o apoio de pessoas denominadas "guardiões". Tratam-se de instrutores de Mindful Eating que foram convidados para colaborar com 
conteúdos relacionados ao tema e veiculados pelo CBME em seu site e suas redes sociais a partir de 2019. Após esse primeiro ano trabalhando em conjunto com o $\mathrm{CBME}$, os guardiões se tornaram membros colaboradores, que são aqueles convidados a atuar de forma mais frequente e efetiva em vários assuntos referentes ao CBME.

Com a expansão do CBME, abriu-se a possibilidade de afiliação. Os membros afiliados não são necessariamente instrutores de Mindful Eating, mas são profissionais que se identificam com o tema e sua forma de abordagem. A afiliação proporciona alguns benefícios exclusivos, especialmente relativos ao acesso aos conteúdos e materiais sobre Mindful Eating oferecidos pelo CBME. Atualmente, este centro é formado por quatro diretoras, quatro membros colaboradores, dois guardiões e 111 membros afiliados.

A estrutura organizacional do CBME é horizontal, descentralizada e baseada no modelo de tecnologia de gestão colaborativa Dragon Dreaming, cuja prática organizativa usa o contexto dialógico de gestão por vias conversacionais e evita o modelo hierárquico piramidal. Tal modelo de gestão busca lidar com a dialética da vida grupal, ao mobilizar as aspirações individuais em favor de um objetivo comum, considerando a inteligência coletiva, o diálogo e os vínculos afetivos ${ }^{(23)}$.

Outro ponto importante de organizações com estrutura horizontalizada entre seus dirigentes é a questão dos diálogos, que acontecem com base nos princípios da Comunicação Não Violenta, que consiste em abordagem específica de comunicação apreciada pelo "falar e ouvir" de forma compassiva, que apoia o estabelecimento de relações de parceria e cooperação. Assim, busca a distinção entre observações e juízos de valor: distinção entre sentimentos e opiniões; necessidades e estratégias; pedidos e exigências ${ }^{(24)}$.

\section{Objetivos e motivações: amor, cuidado e ética}

Dentre os principais objetivos e motivações que permearam a criação de um centro de Mindful Eating no Brasil se destaca a necessidade de construção de um espaço que fosse fonte de informações confiáveis, fomentado por profissionais estudiosos da área e construído a partir das diretrizes internacionais de boas práticas de Mindfulness e Mindful Eating, e que contemplasse tanto o público profissional quanto a população em geral.

$\mathrm{Na}$ contemporaneidade, nota-se situação de "pânico alimentar", especialmente caracterizada pela farmacologização do comer, que supera seus aspectos culturais, sociais e simbólicos. As regras alimentares, pautadas tão somente no papel biológico dos alimentos, são os principais norteadores das escolhas alimentares dos indivíduos, ficando fora das referências o prazer, as preferências e os gostos alimentares das pessoas ${ }^{(25)}$. A frequente prática de dieta como alternativa para se alcançar "saúde" potencializa esse olhar puramente biológico para o comer e a comida, e pode resultar em intensa desconexão com a sinalização interna de fome e saciedade, uma vez que transfere a decisão do que e do quanto comer para regras externas ${ }^{(26)}$. Nesse cenário, emerge o Mindful Eating como alternativa promissora às tradicionais abordagens de dieta, valorizando a alimentação saudável com enfoque no resgate do reconhecimento da sinalização interna do apetite, e na conscientização dos padrões alimentares, sensações corporais e emocionais despertadas pelo comer. Nesse sentido, a mentalidade de dieta, tão comum em nosso meio social e acadêmico, traz a necessidade de pesquisas atualizadas e profissionais especializados em Mindful Eating, para sustentar as propostas dessa abordagem ${ }^{(27-30)}$.

De acordo com o TCME, existem pontos cruciais a serem trabalhados para que haja desenvolvimento adequado das intervenções baseadas em Mindful Eating. Entre eles, destacam-se: reconhecer a natureza multifatorial dos aspectos que envolvem a saúde e o bem-estar; não promover abordagens que tragam controle externo da alimentação - como a prática de dietas; não moralizar a alimentação, e; não promover estigmas de peso e tamanho corporal(31).

Destaca-se também, dentre os objetivos do CBME, o engajamento para a formação de profissionais que desejam se tornar instrutores de Mindful Eating ou incorporar seus conceitos na prática clínica, de forma ética, segura e coerente com as boas práticas internacionais. Assim, o CBME organiza as formações nos protocolos de Mindful Eating e acolhe os futuros instrutores ao promover conteúdos que fomentam a pós-formação.

Acredita-se que a consistência dos protocolos de intervenções baseadas em Mindfulness, que atualmente estão sendo testados, são essenciais para que se possa produzir evidências bem fundamentadas. Esses protocolos trazem como exigência, além do conteúdo de qualificação para formação de um instrutor de Mindfulness, a manutenção e a continuidade de seu desenvolvimento como professor, requisitos importantes e necessários para incorporação do tema na sociedade e na pesquisa científica(32).

A questão ética aplicada ao conceito de Mindfulness e de Mindful Eating emergiu como importante pilar de construção dos objetivos do CBME. 
Em contexto da área da saúde, temos estrutura consolidada e um código ético de conduta profissional baseado na tradição hipocrática, fundamentado nos princípios de respeito pela autonomia, não causar prejuízo e de beneficência, esta última como conceito de fazer o bem ao outro, tanto nas relações como, especialmente, nas condutas profissionais(33). Tais princípios são axiomáticos e fundamentais também para os profissionais que trabalham com os protocolos de atendimento baseados em Mindfulness, estejam eles em ambiente hospitalar ou não. A responsabilidade de trazer conduta ética recai sobre os ombros de cada um que escolhe se engajar como instrutor de intervenções baseadas em Mindfulness, e daí a necessidade de contar com organizações sérias que atuam dentro desses princípios, com intuito de divulgação do tema para a sociedade, networking, formação adequada de instrutores, ao atuar como centro de referência(34).

\section{Desafios e perspectivas futuras: plantar, cuidar e colher}

O CBME vem aprimorando sua estrutura ao longo de sua trajetória, com objetivos e perspectivas que vão sendo construídos e amadurecidos ao longo da caminhada. Durante esses quase quatro anos de existência, conseguiu se estabelecer e se consolidar no Brasil, com o Mindful Eating como sua principal semente a ser cultivada. Trouxe a temática de forma séria, ética e baseada na pesquisa científica, ao criar o site e as redes sociais que são fomentadas frequentemente com informações de qualidade; organizar dois congressos internacionais de Mindful Eating - com mérito de ser o primeiro congresso exclusivamente dedicado à essa temática no mundo; organizar dois retiros de formação presencial, e formar 96 instrutores de Mindful Eating (60 no protocolo Mindfulness-Based Eating Solution - MBES, Eat for Life ; e 36 no protocolo Mindful Eating-Conscious Living - ME-CL).

Para além de cultivar o que foi estabelecido até aqui, existe o interesse em aprimorar o conteúdo científico do CBME com projetos de educação continuada em Mindful Eating, e também trabalhar para que o cuidado nas relações que permeiam o CBME seja sempre prioridade, com interesse em criar e estabelecer um estatuto para guiar os membros dentro dos valores e missões imprescindíveis do Mindfulness e Mindful Eating. Parar, olhar, respirar e degustar cada conquista também é processo importante do CBME, e essencial para a colheita de bons frutos no futuro.

Existe ainda, interesse em trazer para a discussão assuntos que penetram a sociedade em temas culturalmente arraigados, como as questões referentes ao machismo, aos padrões de beleza e lugares ocupados pela mulher na sociedade. A busca por relacionamento saudável com a alimentação e com o corpo também perpassa por esses lugares e, portanto, essa conversa também se faz necessária(35).

\section{Conclusão}

A criação do CBME se deu em momento propício, ao refletir a necessidade de se ter um canal de comunicação para o Mindful Eating, tema muito incipiente no contexto brasileiro. Ao longo de sua construção e estabelecimento como organização, o CBME expressou de forma atenciosa e cuidadosa a importância do cultivo das habilidades de Mindfulness enquanto centro de referência, enquanto profissionais e enquanto instrutores capacitados para atuar na área.

Uma limitação deste trabalho foi não conseguir entrevistar todos os idealizadores da criação do CBME. No entanto, os dados obtidos nessa pesquisa revelaram um importante marco para a história do Mindful Eating no Brasil, ao trazer à tona uma área promissora para a pesquisa científica e saúde coletiva.

A utilização de protocolos baseados em Mindfulness e Mindful Eating em pesquisas científicas se faz necessária para que o tema se fortaleça cada vez mais enquanto importante recurso para saúde e qualidade de vida.

\section{Agradecimentos}

Ao Centro Brasileiro de Mindful Eating por permitir que contássemos sua história.

\section{Referências}

1. Viscardi AAF, Lecuona DS, Correia PMS, Marinho A. A meditação como ferramenta lúdica: potenciais e limites à promoção da saúde. J Manag Prim Health Care [Internet]. 2019 Set; [Acesso 16 abr 2020];100. Disponível em: http://www.jmphc.com.br/jmphc/article/ view/587

2. Tang YY, Lu Q, Feng H, Tang R, Posner MI. Short-term meditation increases blood flow in anterior cingulate cortex and insula. Front Psychol [Internet]. 2015 [cited Jul 10, 2017];6:212. Available from: https://www.ncbi. nlm.nih.gov/pmc/articles/PMC4341506/

3. Dreyfus G. Is mindfulness present-centred and nonjudgmental? A discussion of the cognitive dimensions of mindfulness. Contemporary Buddhism. 2011 Jun; 12(1): 41-54. doi: 10.1080 / 14639947.2011.564815 
4. Eberth J, Sedlmeier P. The Effects of mindfulness meditation: a meta-analysis. Mindfulness. 2012;174-89. doi: $10.1007 /$ s12671-012-0101-x

5. Kabat-Zinn J. Mindfulness. Mindfulness. 2015; 6:1481-83. doi: 10.1007/s12671-015-0456-x

6. Bodhi B. What does mindfulness really mean? A canonical perspective. Contemporary Buddhism. 2011;12(1):19-39. doi: 10.1080 / 14639947.2011.564813

7. Quaglia JT, Braun SE, Freeman SP, McDaniel MA, Brown KW. Meta-analytic evidence for effects of mindfulness training on dimensions of self-reported dispositional mindfulness. Psychol Assess. 2016 Jul; 28(7):803-18. doi: 10.1037/pas0000268

8. Dorjee D. Defining Contemplative Science: The Metacognitive Self-Regulatory Capacity of the Mind, Context of Meditation Practice and Modes of Existential Awareness. Front Psychol. 2016 Nov; 7:1788. doi: 10.3389/fpsyg.2016.01788

9. Pagnini F, Bercovitz K, Langer E. Perceived control and mindfulness: Implications for clinical practice. J Psychother Integr. 2016; 26(2):91-102. doi:10.1037/ int0000035

10. Pagnini F, Langer E. Mindful Reappraisal: Comment on "Mindfulness broadens awareness and builds eudaimonic meaning: a process model of mindful positive emotion regulation". Psychological Inq. 2015 Oct; 26(4):365-7. doi: 10.1080/1047840X.2015.1073660

11. Gethin R. On some definition of Mindfulness. Contemporary Buddhism. 2011 Jun; 12(1):263-79. doi: 10.1080/14639947.2011.564843

12. Framson C, Kristal R, Schenk JM, Littman A J, Zeliadt $S$, Benitez D. Development and validation of the mindful eating questionnaire. J Am Diet Assoc . 2009 Aug;109, 1439-44. doi: 10.1016 / j.jada.2009.05.006

13. Dunn C, Haubenreiser M, Johnson M, Nordby $K$, Aggarwal S, Myer S, Thomas C. Mindfulness Approaches and Weight Loss, Weight Maintenance, and Weight Regain. Curr Obes Rep. 2018 Mar; 7(1):37-49. doi: 10.1007/s13679-018-0299-6

14. Nelson JB. Mindful Eating: The Art of Presence While You Eat. Diabetes Spectr. 2017 Aug; 30(3):171-4. doi: $10.2337 /$ ds $17-0015$

15. Kristeller JL, Wolever RQ, Sheets V. MindfulnessBased Eating Awareness Training (MB-EAT) for binge eating: a randomized clinical trial. Mindfulness. 2014 Jun;5(3):282-97. doi: https://doi.org/10.1007/ s12671-012-0179-1

16. Crane RS, Brewer J, Feldman C, Kabat-Zinn J,Santorelli S, Williams JMG, et al. What defines mindfulness-based programs? The warp and the weft.
Psychol Med. 2016 Apr;47(6):990-9. doi: https://doi. org/10.1017/S0033291716003317

17. Dunne J. Toward an understanding of nondual mindfulness. Contemporary Buddhism. 2011 Jun;12(1):71-88. doi:10.1080/14639947.2011.564820 18. Minayo MCS. O desafio do conhecimento. São Paulo: Hucitec; 2008.

19. Stake RE. Pesquisa qualitativa: estudando como as coisas funcionam. Porto Alegre: Penso; 2011.

20. Bardin L. Análise de conteúdo. 7a ed. São Paulo: Edições 70; 2011.

21. Ribeiro SL. A criação do Centro de Atenção Psicossocial Espaço Vivo. Psicol Ciênc Prof. 2004 Set; 24(3): 92-9. doi:10.1590/S1414-98932004000300012

22. The Center for Mindful Eating [Internet]. About The Center for Mindful Eating [cited Feb 20, 2020]. Available from: https://thecenterformindfuleating.org/ About-TCME

23. Paula APP, Souza MMP. Gestão dialógica e tecnologias colaborativas. Curitiba: Appris; 2018. 131p.

24. Rosenberg MB. Comunicação-não-violenta: técnicas para aprimorar relacionamentos pessoais e profissionais. São Paulo: Ágora; 2006. 288p.

25. Silva AFS, Neves LD, Japur CC, Penaforte TR, Penaforte FR. Construção imagético-discursiva da beleza corporal em mídias sociais: repercussões na percepção sobre o corpo e o comer dos seguidores [Internet]. DEMETRA: Alimentação, Nutrição e Saúde. 2018; 13( 2): 395-411. doi: http://dx.doi.org/10.12957/ demetra.2018.33305

26. Rezende FAC, Penaforte FRO. Dietas e seus desfechos negativos em saúde. In: Rezende FAC, Penaforte FRO, Martins PC. Corpo, comida e comportamento humano. São Paulo: Editora IACI; 2020. p. 19-34.

27. Bush HE, Rossy L, Mintz LB, Schopp L. Eat for Life: a work site feasibility study of a novel mindfulness-based Intuitive Eating intervention. Am J Health Promot. 2014 Aug;28(6):380-8. doi:10.4278/ajhp.120404-QUAN-186 28. Wansink B, Sobal J. Mindless Eating: the 200 daily food decisions we overlook. Environ Behav. 2007 Jan;39(1):106-23. doi: 10.1177 / 0013916506295573 29. Albers S. Using Mindful Eating to treat restriction: a case study. Eat Disord. 2011 Jan-Feb; 19:97-107. doi: 10.1080 / 10640266.2011 .533609

30. Daly P, Pace T, Berg J, Menon U, Szalacha LA. A Mindful Eating intervention: A theory-guided randomized anti-obesity feasibility study with adolescent Latino females. Complement Ther Med. 2016 Oct; 28:22-8. doi: 10.1016 / j.ctim.2016.07.006.

31. The Center for Mindful Eating [Internet]. Position Statements on Topics Related to Mindful 
Eating [cited Feb 20, 2020]. Available from: https:// thecenterformindfuleating.org/Position-Statements

32. Gherardi-Donato ECS. O Mindfulness Trainings International (MTi) e a formação de instrutores de mindfulness no Brasil. SMAD, Rev Eletr Saúde Mental Álcool Drog [Internet]. 2018 [cited Mar 9, 2020];14(2):62-4. Available from: http://www.revistas. usp.br/smad/article/view/151310

33. Beauchamp TL, Childress JF. Princípios de ética biomédica. 4a ed. São Paulo: Loyola; 2002.

34. Kabat-Zinn J. Too early to tell: the potential impact and challenges - ethical and otherwise - inherent in the mainstreaming of dharma in an increasingly dystopian world. Mindfulness. 2017 Jun; 8:1125-35. doi:10.1007/ s12671-017-0758-2

35. Pinto CRJ. Feminismo, história e poder. Rev Sociol Polít. 2010 Jun;18 (36):15-23. doi: 10.1590/S010444782010000200003 .

\section{Contribuição dos autores}

Concepção e planejamento do estudo: Ana Paula Leme de Souza e Driele Cristina Gomes Quinhoneiro. Obtenção dos dados: Ana Paula Leme de Souza. Análise e interpretação dos dados: Ana Paula Leme de Souza. Obtenção de financiamento: Ana Paula Leme de Souza.

Redação do manuscrito: Ana Paula Leme de Souza e Fernanda Rodrigues de Oliveira Penaforte. Revisão crítica do manuscrito: Ana Paula Leme de Souza, Fernanda Rodrigues de Oliveira Penaforte, Lívia Dayane de Sousa Azevedo, Driele Cristina Gomes Quinhoneiro e Rosane Pilot Pessa.

Todos os autores aprovaram a versão final do texto.

Conflito de interesse: os autores declararam que não há conflito de interesse.
Recebido: 10.03.2020

Aceito: 22.05 .2020 